\title{
EDITORIAL
}

\section{HOMENAJE A LA DRA. MARIA MAGDALENA SCHIAVONE}

Conocí a la Dra. María Magdalena Schiavone - "Magui" como la conoce todo el mundo - en el examen final de Introducción a la Botánica, una materia de primer año en la que ella formaba parte del tribunal. Terminé de rendir y me dijo ¿porque no te pasas a botánica?; al año siguiente empecé el cursado de su materia y, desde entonces, el vínculo maestro-discípulo se creó y fue fortaleciéndose rápidamente. La recuerdo siempre con alegría, ponderando la pasión que la caracteriza, tanto para retarme cuando salían mal los cortes como para transmitirme el amor por las briofitas.

La Dra. Schiavone obtuvo el título de Licenciada en Ciencias Biológicas orientación Botánica en el año 1972 en la Facultad de Ciencias Naturales de la Universidad Nacional de Tucumán (UNT), alcanzando el grado académico de Doctora en Ciencias Biológicas en octubre de 1976 en la misma universidad. Su tesis doctoral versó sobre Polytrichaceae del noroeste de Argentina, aunque tiempo más tarde extendió sus estudios al resto del país. Los artículos surgidos de este trabajo siguen siendo un punto de referencia para los estudiosos de la subclase Polytrichidae.

En el año 1973, ingresó como Auxiliar Docente de primera en la Facultad de Ciencias Naturales de la UNT, en donde trabajó de forma activa en diferentes cargos y cátedras de botánica hasta el año 2016. En el transcurso de este prolongado periodo dictó decenas de cursos de grado y posgrado tanto en la Argentina como en el resto del mundo hispano hablante. Constituyó cientos de tribunales examinadores para evaluación de estudiantes, concursos docentes, tesis de grado y posgrado.

La gestión universitaria realizada por Magui fue extensa, siendo consejera en varios periodos, pero se destaca su accionar como Vicedecana (1996-1998) y su compromiso como Secretaria Académica por dos periodos consecutivos (1998-2006) en la Facultad de Ciencias Naturales e Instituto Miguel Lillo.

La gran preocupación de Magui fue siempre la formación de recursos humanos, trabajó arduamente entregando en forma generosa y desinteresada sus conocimientos a una veintena de tesinistas, tesistas, becarios y pasantes. Asimismo, fue directora de diferentes programas y proyectos de investigación subsidiados por CONICET, CIUNT y PIUNT, investigando las briofitas en los diferentes contextos de la vegetación vascular y, a su vez, a la Flora Criptogámica en general junto a Celina Matteri y Gabriela Hässel de Menéndez. Trabajando en la Fundación Miguel Lillo como jefa del laboratorio de briología y como fruto de los proyectos que dirigió, público numerosos artículos sobre la flora de musgos y hepáticas de Argentina y Latinoamérica que le sirvieron para obtener reconocimiento por parte de la comunidad botánica argentina e internacional.

Junto a Celina Matteri y Gabriela Hassel de Menéndez constituyen los máximos exponentes de la briología argentina contemporánea. Por todo esto - sus logros, su entrega, su compromiso y su pasión realizamos este volumen en homenaje a nuestra querida Dra. María Magdalena Schiavone...Magui.

Guillermo M. Suárez

\section{AnécDotas y Salutaciones}

A primeira vez que encontrei Magui ela estava sentada junto com Celina Matteri tomando um café no restaurante de um hotel na cidade do México onde aconteceu um Simpósio da Socieda de Latinoamericana de Briologia. As duas me viram sozinha e perguntaram se eu iria participar do encontro e me convidaram para sentar com elas. Nesse dia começou uma linda e duradoura amizade. Sempre que nos encontramos pelo mundo os momentos são maravilhosos em todos os sentidos porque a sabedoria e a alegria de Magui são realmente contagiantes e alegram o coração de quem tem a felicidade de ter sua amizade.

Um abraço,

Denise Pinheiro da Costa 
Magui en el laboratorio es toda la inspiración que uno necesita para seguir adelante en el camino de las briofitas. Cada mañana, durante mis pasantías, su primer paso en el laboratorio venía acompañado de un “yo creo que lo que vos tenés ahí es...”.Así empezábamos, desde el minuto cero a trabajar. Cada vez que empiezo una determinación, se me vienen sus palabras "si tiene papilas, tiene papilas, si tiene mamilas tiene mamilas", dándome el empuje necesario para aclarar mi cabeza de que es lo que estoy viendo. En mi última pasantía, recuerdo que compartimos muchos momentos, en su rinconcito del laboratorio, ella leyendo en voz alta para ambas, mientras intentábamos entender que era ese fósil que estábamos estudiando. Esos dulces momentos y todo su carácter y sinceridad, que cuando lo que uno hace es una porquería lo dice, lo llevo conmigo para siempre. Te agradezco Magui, sinceramente, todas tus enseñanzas y te admiro profundamente.

Adolfina Savoretti

Como estudiante de grado, conocí a la Dra. Magui en el cursado de la materia "Diversidad Vegetal I" -aunque en ese momento me refería a ella simplemente como "Profe". Ya en ese entonces la destacaba su estilo de docencia tan particular. No sólo tenía (y ciertamente aún tiene) un conocimiento casi enciclopedista del mundo briológico, sino que también era dueña (y por supuesto, aún lo es) de un carisma excepcional. Cada comentario, cada pensamiento "en voz alta”, transmitía pasión además de conocimiento. Así fue que por aquellos años Magui se convirtió en una de las docentes a quien más respeto y admiración supe guardar. Años después, finalizando mis estudios de grado y comenzado los de posgrado, comencé a trabajar en el área de la briología; motivo por el cual, Magui -quien pasaría a ser de ahora en más "Dra. Magui”- sería fundamental para dar mis primeros pasos. Más allá de cualquier tipo de lección técnica, la Dra. -muy probablemente sin darse cuenta- me enseñó el valor del esfuerzo cotidiano. Desde su lugar como docente recuerdo sus sugerencias a los estudiantes para cuidar su desempeño. Desde su lugar como jefa de laboratorio recuerdo sus sugerencias para mejorar la calidad de los trabajos. Recuerdo, con especial cariño, esas ocasiones que se daban naturalmente durante los viajes de campo: todos alrededor suyo escuchando sus anécdotas sobre tal o cual campaña, sobre tal o cual especie, o sobre tal o cual hallazgo. Magui, por todo lo enseñado y transmitido, tus discípulos te estaremos siempre agradecidos.

Jorge R. Flores

Me alegra mucho poder participar en este homenaje a Magui Schiavone, quien fue una de las personas que me motivó a dedicarme a la briología cuando recién comenzaba a trabajar con musgos. Nunca olvidaré esas horas que pasamos juntos en el hospital público de La Serena cuando lamentablemente sufrió un accidente en la salida al campo del congreso latinoamericano del año 2010. Después de una larga espera Magui salió con un hermoso yeso en su pierna, regalo del pueblo chileno.

Juan Larrain

Conocí a la Dra. Magui un par de años antes de recibirme, cuando comencé las pasantías en Tucumán bajo su dirección, para iniciar mis estudios de Bryophyta con un género muy particular (Sphagnum), con el cual casualmente la Dra. había trabajado y coleccionado mucho en sus viajes por la Patagonia, a la par de la Dra. Matteri. Recuerdo que una tarde, cuando hablábamos de los colores de Sphagnum en el campo, la Dra sacó un álbum grande de diapositivas, y a contraluz me mostró las fotos de ella y la Dra Matteri en las turberas, mientras relataba con memoria exacta, y pasión a flor de piel, anécdotas de esos viajes que tanta experiencia le dieron. Esa tarde, la Dra. en sus relatos, me enseñó no sólo el valor de los viajes de campo para la experiencia de un briólogo. También me mostró que el motor principal de este trabajo es esa pasión que ella le imprime a todo lo que hace (incluso para retarme!), y para mí fue una actitud digna de imitar. Los buenos maestros enseñan más allá del contenido... los buenos maestros inspiran. Gracias Dra.!

Soledad Jimenez 

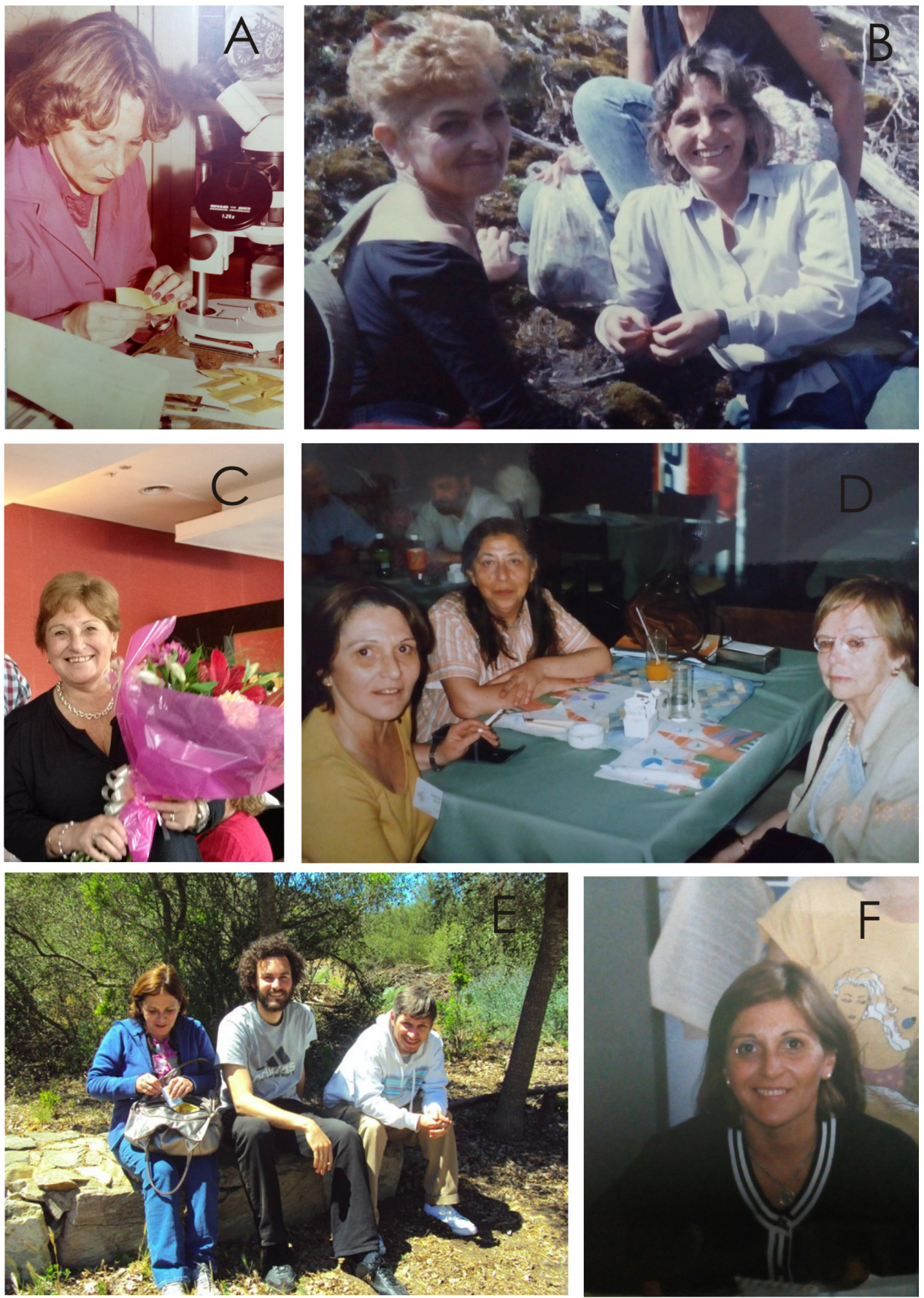

A. Magui Schiavone durante la realización de su tesis doctoral. B. Magui en un viaje de colección junto a su amiga Celina Matteri. C. Magui en el día de su jubilación D. Magui junto a Carolina Villagran y Gabriela Hassel de Menendez en el marco de la JAB en San Luis. E. Magui junto a Juan Larraín y Guillermo Suárez en el congreso Internacional de Botánica en la Serena, Chile. F- Magui en su laboratorio. 
